\title{
Quantization-aware Sensor Selection for Source Localization in Sensor Networks
}

\author{
Yoon Hak Kim, Member, KIMICS
}

\begin{abstract}
In distributed source localization where sensors transmit measurements to a fusion node, we address the sensor selection problem where the goal is to find the best set of sensors that maximizes localization accuracy when quantization of sensor measurements is taken into account. Since sensor selection depends heavily upon rate assigned to each sensor, joint optimization of rate allocation and sensor selection is required to achieve the best solution. We show that this task could be accomplished by solving the problem of allocating rates to each sensor so as to minimize the error in estimating the position of a source. Then we solve this rate allocation problem by using the generalized BFOS algorithm. Our experiments demonstrate that the best set of sensors obtained from the proposed sensor selection algorithm leads to significant improvements in localization performance with respect to the set of sensors determined from a sensor selection process based on unquantized measurements.
\end{abstract}

Index Terms - quantization, sensor selection, rate allocation, source localization

\section{INTRODUCTION}

ONE important sensor collaboration task with broad applications is source localization. The goal is to estimate the location of a source within a sensor field, where a set of distributed sensors measures the acoustic or seismic signals emitted from a source and manipulates the measurements to produce meaningful information such as signal energy, direction-of-arrival (DOA) and time difference-of-arrival (TDOA) $[1,6]$ which are then transmitted to a fusion node where an estimate of the source location is produced.

The localization performance improves with an increasing number of measurements from sensors which will require many sensors involved in localization. Collecting measurements from many sensors and transmitting them for localization will reduce the lifetime of the network. Thus sensing tasks (e.g., localization, tracking targets) should be able to minimize the number of sensors involved in order to

\footnotetext{
Manuscript received February 19, 2011; revised April 4, 2011; accepted April 15, 2011.

Yoon Hak Kim is with System LSI Division, Samsung Electronics, Giheung campus, Gyeonggi-Do, Korea 446-711 (email: yhk418@, gmail.com)
}

prolong the system lifetime. This will motivate the selective use of informative sensors and reduce the number of sensors needed. In the scenario of localization using wireless sensor networks, the localization accuracy can be significantly improved by selecting the most informative sensors.

Previously, an entropy based heuristic approach proposed in [10] greedily selects the next sensor to reduce overall uncertainty. The authors in [2] focus on finding the best set of sensors that maximizes localization accuracy under the assumption of known uncertainty region for source location and present analytical bounds on the performance of their sensor selection algorithm. Note that none of these approaches takes into account quantization of sensor measurements and quantization techniques used in each sensor. Since practical systems will require quantization of the measurements before transmission, the quality of localization ought to be evaluated based on quantized measurements. In [7,8], the authors addressed ML (Maximum Likelihood) estimation problem for source localization using quantized data and derived CramerRao Bound (CRB) for comparison. Especially, in [7] they also considered heuristic quantization schemes.

In this paper, we address the sensor selection problem when quantization of measurements is taken into account. For this case, it should be observed that different rates at sensors will lead to different sensor selection solutions. Without quantization, assuming the same sensor characteristics, sensor locations have been crucial factors for this optimization problem. However, when quantization is involved, rates assigned to sensors and quantization schemes will also make critical impacts on the optimization solution.

We show that this quantization aware sensor selection problem can be treated as one of the rate allocation problems where the goal is to allocate the rate to each sensor so as to minimize the localization error. To solve the problem we apply the generalized BFOS (GBFOS) algorithm (see [9]) which requires calculation of Rate-Distortion (R-D) points for each candidate rate allocation. Then we demonstrate that the best sensor selection can be achieved by jointly optimizing both rate allocation and sensor selection. In addition, since power consumption due to rate transmission to the fusion node is proportional to the distance between each sensor and the fusion node, the same rate at different sensors may lead to different 
power consumption and thus to different sensor selection. With this consideration, we also address the sensor selection problem with power constraint where our goal is to find the best set of sensors that achieves optimal localization accuracy for a given power consumption.

Throughout the paper, it is assumed that each sensor can obtain the noise-corrupted measurement such as signal energy, or DOA using actual measurements (e.g. time-series measurements or spatial measurements) and there is only one way noiseless communication channel from sensors to fusion node, i.e., there is no feedback channel and the sensors do not communicate with each other (no relay between sensors).

Our experiments show that rates should be distributed so as to lead to partition of the sensor field that is as uniform as possible. Thus, when several sensors are clustered together, the rate per sensor tends to be lower than when the same sensors are more spread out. They also show that our sensor selection optimized via rate allocation process allows us to achieve significant improvement in localization accuracy as compared with sensor selections obtained by an exhaustive search where the quality of localization is evaluated based on unquantized measurements.

This paper is organized as follows. The problem formulation is given in Section II where the localization system is briefly introduced and application of the generalized BFOS algorithm to rate allocation problem is explained. In Section III, the sensor selection problem is formulated as one of rate allocation problems. Simulation results are given in Section IV and the conclusion is found in Section V.

\section{PROBLEM FORMULATION}

Within the sensor field $S$ of interest, suppose that there are $M$ sensors located at known spatial locations, denoted $x_{i}, i=1, \ldots, M \quad$, where $x_{i} \in S \subset R^{2}$. The sensors measure signals generated by a source assumed to be located at an unknown location $x \in S$ and to be static during the localization process. We assume that the i-th sensor measures the source signal over a time interval $k$. This measurement is denoted by $z_{i}$ with:

$$
z_{i}(x, k)=f_{i}\left(x, x_{i}\right)+\omega_{i}(k)
$$

where $f_{i}\left(x, x_{i}\right)$ indicates the sensor model employed at sensor $i$ and $\omega_{i}$ is a combined noise term that includes both measurement noise and modeling error and can be approximated using a normal distribution, $N\left(0, \sigma_{i}^{2}\right)$. It is assumed that each sensor measures its sensor reading $z_{i}(x, k)$ at time interval $k$, quantizes it and sends it to a fusion node, where all sensor measurements are used to obtain an estimate $\hat{x}$ of the source location. Suppose that at sensor $i$ we use a $R_{i}$-bit quantizer with a dynamic range of $\left[z_{i, \text { min }}\right.$ $\left.z_{i, \max }\right]$. We assume that the quantization range can be selected for each sensor based on desirable properties of their respective sensing ranges.

We believe that this formulation is general and captures many scenarios of practical interest. For example, $z_{i}(x, k)$ could be the energy captured by an acoustic amplitude sensor but it could also be a DOA measurement. (In the DOA case each measurement at a given location will be provided by an array of colocated sensors.) Each scenario will obviously lead to a different sensor model $f_{i}\left(x, x_{i}\right)$. We assume that the fusion node needs measurements, $z_{i}(x, k)$ from all sensors in order to estimate the source location. In some cases, one measurement per sensor is used, while in other cases values of $z_{i}(x, k)$ for several $k$ are needed for localization. Notice that when there are multiple measurements at each sensor, all individual measurements need not be sent. Instead, a sufficient statistic for source location, $x$ can be computed, quantized and transmitted. It can be easily shown that the average of the measurements at each sensor is a sufficient statistic for $x$. This method can be justified by noting the fact that there would be no degradation in localization accuracy if localization is performed based on the averages sent from each sensor, while reducing multiple measurements into a single value (say, average) to be quantized.

In what follows we discuss the design of the localization system, including the localization function operating on quantized data, quantization schemes and the algorithm to assign different rates to different sensors.

\section{A. Location Estimation based on Quantized Data}

Clearly, for $z_{i}(x, k)$ to be useful for localization, it must be a function of the relative positions of the source and the sensor. Thus, there exists some function $g_{u}($.) that can provide an estimate of the source location $\hat{x}$ based on the original, unquantized measurements; these estimators have been the focus of most of the literature to date, for both sensor networks and other source localization scenarios. Instead, we consider the corresponding estimators $g($.) that operate on quantized data to estimate the source location $\hat{x}$. 
While specific $g($.$) choices depend on the sensor$ model $f_{i}\left(x, x_{i}\right)$, we can sketch some of the general properties of this estimator First, since $z_{i}(x, k)$ is used for localization, it must provide information (distance, angle, etc) about the relative position of sensor and source. Thus, after quantization, each transmitted symbol will represent a range of positions (e.g. a range of distances from the sensor or an angular range). Second, once information is obtained from all sensors, it would be optimal to exploit the range information corresponding to each quantized measurement without obtaining its reconstructed value which has been used in a standard estimator (i.e., replacing a range of distances by a single distance). That is, an optimal estimator $g($.) should be a function of range information rather than reconstructed values. Our previous work [4] explores optimal estimators in minimum mean square error (MMSE) sense which use the range information.

\section{B. Quantization Schemes}

We discuss two different quantization schemes in this section.

- Uniform quantizer: simplest form of quantization. Due to its simplicity, it has been widely used in many literatures. However, it would lead to poor sensor selections since this quantization does not exploit information about sensor locations and sensor characteristics.

- Geometry-driven quantizer: sensor characteristics can be efficiently used to produce this quantizer. For example, in an acoustic amplitude sensor case, it is designed so as to partition uniformly the distance between sensors and source. Equally Distance-divided Quantizers (EDQ) are introduced and shown to be simple and achieve good performance [3]. Similar ideas can be applied to DOA sensors, where quantizers provide uniform quantization with respect to the angle of arrival. Clearly, since this quantizer is designed by taking into account sensor characteristics, it would yield a good sensor selection.

\section{Rate Allocation}

Given total rate $R_{T}=\Sigma R_{i}$, M quantizers and the estimate, $\hat{x}$ (equivalently, $g($.$) ), the goal is to find the rate$ allocation, $R^{*}=\left(R_{1}, \ldots, R_{M}\right)$ that minimizes the average localization error given by $D=\int_{x \in S}\|x-\hat{x}\|^{2} p(x) d x$ where $p(x)$ is the distribution of source location $x$.

A more general problem formulation can take into consideration transmission costs, i.e., the power consumption in the network required to transmit bits to the fusion node. This power consumption will depend on the bit allocation to specific sensors but also on the distance from these sensors to the fusion node. Thus, we can address the rate allocation problem with power constraint as follows: we are given a total power, $P=\Sigma_{1}^{M} P_{i}=\Sigma_{i} C_{i} R_{i}$ where $C_{i}$ is the power required for sensor $i$ to transmit one bit to a fusion node, $x_{f}$ and thus $P_{i}$ the power consumption at sensor $i$. Our goal is then to find the rate allocation $R^{*}$ that minimizes the average localization error for a given total power.

Clearly, $C_{i}$ is proportional to the physical distance between $x_{i}$ and $x_{f}$ and thus once the sensors are deployed in a sensor field, it can be determined prior to the rate allocation. $C_{i}$ can be written as $C_{i}=\gamma_{i}\left\|x_{f}-x_{i}\right\|^{\alpha}$ where $\alpha$ is the exponent for path-loss and $\gamma_{i}$ reflects transmission method and other factors [11]; in this paper $\gamma_{i}$ is assumed to be equal for all sensors and thus ignored for simplicity. Notice that only the relative values for $C_{i}$ will play a role in this rate allocation process.

To solve the rate allocation problem for source localization, we can apply the well-known Generalized BFOS algorithm (GBFOS) [9] to obtain $R^{*}$. Initially, $R_{i}=R_{T}$ and at each iteration we reduce the rate allocated to one of the sensors by computing alternative rate-distortion (R-D) operating points for each candidate rate allocation and choosing the one that minimizes the slope of the R-D curve. This is done repeatedly until $\sum_{i} R_{i}=R_{T}$ is satisfied. Formally, at the $\eta$-th iteration,

$$
i^{\eta}=\operatorname{argmin}_{1 \leq i \leq M} \frac{D_{i}(\eta)-D(\eta-1)}{\Delta R_{i}^{\eta}}
$$

where $D(\eta-1)$ is the average localization error at the previous step, $\quad D_{i}(\eta)=\int_{i \in S}\left\|x-\hat{x}^{i}(\eta)\right\|^{2} p(x) d x, \quad \hat{x}^{i}(\eta)$ is computed using $g($.$) and \mathrm{M}$ quantizers are assumed to be given with $R^{i}=\left(R_{1}^{\eta}=R_{1}^{\eta-1}, \ldots, R_{i}^{\eta}=R_{i}^{\eta-1}-\Delta R_{i}^{\eta}, \ldots, R_{M}^{\eta}=R_{M}^{\eta-1}\right) \quad$. The GBFOS algorithm can also be applied to the rate allocation problem with power constraints. Similarly, at each step we reduce the power allocated to individual sensors until $\Sigma_{i} C_{i} R_{i}=P$ is satisfied. Formally, at the $\eta_{t h}$ iteration,

$$
i^{\eta}=\arg \min _{1 \leq i \leq M} \frac{D_{i}(\eta)-D(\eta-1)}{\Delta P_{i}^{\eta}}
$$

where $\Delta P_{1}^{\eta}=C_{i} \Delta R_{i}^{\eta}$. 


\section{SENSOR SELECTION PROBLEM}

In this section, we consider the sensor selection problem where the goal is to find the best set of sensors that maximizes localization accuracy while taking into account quantization of sensor measurements. Here, we formulate this as follows: we are given a set $V=\left\{s_{1}, \ldots, s_{M}\right\} \quad$ of $\mathrm{M}$ sensors deployed in a sensor field $S$, and we are also given the subset $A_{S}$ of $S$ and distribution $p(x)$ of source location where $\int_{i \in A_{S}} p(x) d x=1$. Note that $A_{S}$ may be a subset of $S$ or $S$ itself, depending on the applications. The goal is, given the total $\operatorname{cost} C_{T}$, to select the best set of sensors such that the average localization error computed using quantized measurements is minimized, with constraints on the total cost. Formally, we have

$$
V^{*}=\operatorname{argmin}_{V^{\prime}, C\left(V^{\prime}\right) \leq C_{T}} \int_{t \in A_{S}}\left\|x-\hat{x}_{V^{\prime}}\right\|^{2} p(x) d x
$$

where $V^{\prime}$ is one of possible subsets of $V$ and $C_{T}$ can be total rate, the number of sensors or/and power consumption. $C\left(V^{\prime}\right)$ is the cost function computed over $V^{\prime}$ and $\hat{x}_{V^{\prime}}$ is obtained using the quantized sensor measurements from sensors in $V^{\prime}$.

In the previous section, we have focused on finding the rate allocation that minimizes the average localization error, given the total rate or power consumption. Thus once the optimal solution is obtained, it could afford the solution to the sensor selection problem with the same total cost since it also minimizes the average localization error given by (4). That is, the optimal set $V^{*}$ can be constructed as a set of the sensors in the optimal rate allocation, $R^{*}$ with nonzero bits assigned. In case of power consumption, there are some factors that should be taken into account in order to solve the sensor selection problem by the rate allocation with power constraint. For example, there will be the power, $P_{r}$ for activating sensors which has not been considered in our rate allocation process. That is, $P_{i}$ should be replaced by $P_{i}=P_{r}+C_{i} R_{i}$. Obviously the sensor selection problem can be considered as one of the rate allocation problems and can be directly solved by obtaining $R^{*}$. Notice there will be some cases that require some modification to our rate allocation process in order to obtain the optimal solution to the sensor selection problem. For example, when $C_{T}$ is a combined cost of the total rate $C_{R}$ and the total number of sensors $C_{N}$, the rate allocation process with $R_{T}=C_{T}$ will be conducted to find the optimal rate allocation $R^{*}$.
However, if $V^{*}$ constructed from $R^{*}$ contains more sensors than the cost $C_{N}$, the process should continue further by constructing all possible candidate sets of $C_{N}$ sensors selected from $V^{*}$ and allocating the rate deprived of the unselected sensors in $V^{*}$ to $C_{N}$ sensors in each constructed set such that one of the candidate sets minimizing the localization error would afford the solution to the sensor selection problem.

\section{IV.SIMULATION RESULTS}

In the experiments, our sensor selection algorithm is verified for an acoustic amplitude sensor system where each sensor adopts the energy-decay sensor model proposed in [5]. It is assumed that each sensor uses EDQ (geometry-driven quantizer) during the sensor selection process except for the experiments where otherwise stated. In performing the localization based on quantized sensor measurements, the localization algorithm proposed in the previous work [4] is applied to obtain the localization function $g($.$) . Experimental results are$ evaluated in terms of the average localization error (LE), $E\left(\|x-\hat{x}\|^{2}\right)$ computed using EDQ.

\section{A. Sensor selection and rate allocation}

For the two 5-sensor configurations in Figure 1, sensor selection with $C_{T}$ equal to a combined cost of $C_{N}=3$ sensors and $C_{R}=10$ bits is performed by taking three different strategies for the purpose of performance evaluation. First, under the assumption of unquantized sensor measurements, sensor selection process with $C_{N}=3$ sensors is conducted to find the best set $V_{U Q}^{*}$ by using an exhaustive search over $V=\{1,2,3,4,5\}$ where $\hat{x}_{V^{\prime}}$ in (4) is computed based on unquantized measurements. After $V_{U Q}^{*}$ is found, rate allocation over 3 sensors in $V_{U Q}^{*}$ with $R_{T}=C_{R}=10$ bits described in Section II-C is executed to produce $R_{U Q}^{*}$. Second, it is assumed that we are given a fixed rate allocation $R_{f i x}$ randomly chosen for $C_{N}=3$ sensors which satisfies $C_{R}=\Sigma R_{i}=10 \quad$ (e.g., $\left.\left[\begin{array}{lll}3 & 4 & 3\end{array}\right]\right)$ and sensor selection is processed to find the best set $V_{f i x}^{*}$ by the same exhaustive search in which $\hat{x}_{V^{\prime}}$ in (4) is obtained based on quantized measurements generated from the given rate allocation $R_{f i x}=\left[\begin{array}{lll}3 & 4 & 3\end{array}\right]$ at each iteration. 


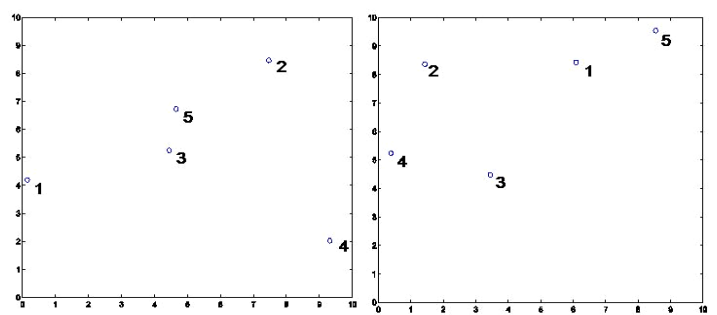

Fig. 1. Two 5-sensor configurations: 5 acoustic amplitude sensors are deployed in a sensor field $\left(10 \times 10 \mathrm{~m}^{2}\right)$, respectively.

Rate allocation over the sensors in $V_{f x}^{*}$ is also carried out to yield $R_{f i x}^{*}$. Finally, the proposed algorithm for sensor selection is performed to attain the solution $V^{*}$ (equivalently, $R^{*}$ ) via the rate allocation in Section IIC. Note that the proposed algorithm allows us to achieve joint optimization of rate allocation and sensor selection. The experimental results for the two configurations are provided in Table I and II, respectively where the localization errors are computed using EDQs designed for several different rate allocations respectively. It should be pointed out that 1) $V_{U Q}^{*}$ considered to be optimal for unquantized measurements is no longer the best set when quantization is taken into account and 2) rates assigned to each sensor is another crucial factor as well as sensor locations, in determining the best set. Clearly, the best strategy would be to optimize rate allocation and sensor selection jointly.

From a 5-sensor configuration (left) in Figure 1, it can be said that sensors 3 and 5 are so close to each other that they provide redundant information for localization and thus the optimal solution allocates few bits to both these sensors. In fact, in our example, at relatively low rates (an average of 2 bits per sensor) it is more efficient to send information from only three sensors (sensor 1,2 and 4), i.e., allocating zero bits for the other two sensors (sensor 3 and 5). From the optimal rate allocation $R^{*}=\left[\begin{array}{lllll}4 & 3 & 0 & 3 & 0\end{array}\right]$, we can construct a set of the sensors (sensor 1,2 and 4), $V^{*}$ with nonzero bits, which corresponds to the solution for the sensor selection problem in Section III that minimizes the localization error. Similar interpretation can be provided for the other 5-sensor configuration (right) in Figure 1. To check the effect of different quantization schemes at each sensor, the proposed algorithm is also conducted using uniform quantizers at each sensor to produce $V_{U}^{*}$ (equivalently, $R_{U}^{*}$ ). This experiment shows that good quantization scheme (in this case, geometry-driven quantizers) is needed to achieve a good sensor selection as compared with uniform quantizers.
For the configuration (left) in Figure 1, our sensor selection solution $V^{*}$ is compared with $V_{U Q}^{*}$ in a noisy case (see Table III). The average localization error is computed with $\sigma_{i}=\sigma=0.05$. Clearly, although our sensor selection solution $V^{*}$ is obtained in a noiseless condition $\left(\omega_{i}=0\right.$ in (1)), it also provides improved localization accuracy even when the measurements are noisecorrupted.

TABLE I

LOCALIZATION ERROR (LE) FOR FIGURE 1 (LEFT)

\begin{tabular}{|c|c|c|}
\hline Best set of sensors & Rate allocations & $\operatorname{LE}\left(\mathrm{m}^{2}\right)$ \\
\hline$V_{U Q}^{*}=\{1,3,5\}$ & $R_{U Q}^{*}=\left[\begin{array}{lll}3 & 3 & 4\end{array}\right]$ & 0.6086 \\
\hline$V_{U}^{*}=\{1,4,5\}$ & $R_{U}^{*}=\left[\begin{array}{lllll}6 & 0 & 0 & 3 & 1\end{array}\right]$ & 1.2360 \\
\hline$V^{*}=\{1,2,4\}$ & $R^{*}=\left[\begin{array}{lllll}4 & 3 & 0 & 3 & 0\end{array}\right]$ & 0.1522 \\
\hline
\end{tabular}

TABLE II

LOCALIZATION ERROR (LE) FOR FIGURE 1 (RIGHT)

\begin{tabular}{|c|c|c|}
\hline Best set of sensors & Rate allocations & $\mathrm{LE}\left(\mathrm{m}^{2}\right)$ \\
\hline$V_{i Q}^{*}=\{1,2,3\}$ & $R_{v_{Q}}^{*}=\left[\begin{array}{lll}4 & 3 & 3\end{array}\right]$ & 0.2826 \\
\hline$V_{f i x}^{*}=\{2,4,5\}$ & $R_{f x x}^{*}=\left[\begin{array}{lll}4 & 2 & 4\end{array}\right]$ & 0.1808 \\
\hline$V^{*}=\{2,3,5\}$ & $R^{*}=\left[\begin{array}{lllll}0 & 4 & 2 & 0 & 4\end{array}\right]$ & 0.1600 \\
\hline
\end{tabular}

TABLE III

LOCALIZATION ERROR (LE) FOR TWO DIFFERENT SENSOR SELECTION IN NOISY CASE $\sigma=0.05$

\begin{tabular}{|c|c|c|}
\hline Best set of sensors & Rate allocations & $\mathrm{LE}\left(\mathrm{m}^{2}\right)$ \\
\hline$V_{U Q}^{*}=\{1,2,3\}$ & $R_{U Q}^{*}=\left[\begin{array}{lll}3 & 4 & 3\end{array}\right]$ & 0.8018 \\
\hline$V^{*}=\{1,2,4\}$ & $R^{*}=\left[\begin{array}{llll}4 & 3 & 0 & 3\end{array}\right]$ & 0.2587 \\
\hline
\end{tabular}

\section{B. Sensor Selection with Power Constraints}

The sensor selection with power constraints is executed using EDQs for the same sensor configuration as in Figure 1 (left). In this experiment, the fusion node, $x_{f}$ is assumed to be located at $(10,10)$ and the power consumption at sensor $\mathrm{i}$ is also assumed to be computed by $P_{i}=C_{i} R_{i}=\left\|x_{f}-x_{i}\right\|^{\alpha} R_{i}$ where $\alpha=1$ for simplicity. The optimal sensor selection $V_{P W}^{*}$ (equivalently, $R_{P W}^{*}$ ) is produced with $C_{T}$ given by the total power (in this experiment, $C_{T} \approx 70$ ) which is equal to the power consumed by 5 sensors when each sensor uses $R_{i}=2$ bits. The test results for $V_{P W}^{*}, V^{*}$ and a uniform rate allocation $R^{U}$ are provided for comparison in Table IV. It can be seen that the sensors (sensors 2,4 and 5) closer to $x_{f}$ will be assigned higher rate and sensor 3 will be allocated 
lower rate since it would provide redundant information along with sensor 5 . Note that significant performance gain is observed as compared with the system $\left(R^{U}=[222\right.$ 2 2]) that consumes the same power. Obviously, the sensor selection $V^{*}=\{1,2,4\}$ optimized under equal power assumption $\left(C_{i}=\right.$ constant $)$ is no longer the optimal one for the sensor selection problem with power constraint.

\section{TABLE IV}

\section{LOCALIZATION ERROR (LE) FOR SENSOR SELECTION SOLUTIONS WITH POWER CONSTRAINT}

\begin{tabular}{|c|c|c|}
\hline Best set of sensors & Power & $\mathrm{LE}\left(\mathrm{m}^{2}\right)$ \\
\hline 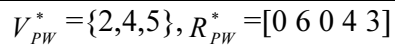 & 68.5841 & 0.0155 \\
\hline$V^{*}=\{1,2,4\}, R^{*}=\left[\begin{array}{lllll}4 & 3 & 0 & 3 & 0\end{array}\right]$ & 78.6355 & 0.1533 \\
\hline$R^{U}=\left[\begin{array}{lllll}2 & 2 & 2 & 2 & 2\end{array}\right]$ & 71.9401 & 0.6199 \\
\hline
\end{tabular}

\section{Performance Evaluation}

In the experiments, the proposed sensor selection is further conducted for 100 different sensor configurations to compute average performance gain. The experimental results are tabulated in Table V. As expected, our joint optimization algorithm for rate allocation and sensor selection shows significant improvement in localization accuracy as compared with a typical sensor selection solution $V_{U Q}^{*}$.

\section{TABLE V \\ LOCALIZATION ERROR (LE) FOR TWO DIFFERENT SENSOR SELECTION SOLUTIONS}

\begin{tabular}{|c|c|c|}
\hline Sensor selection solution & Rate allocations & $\mathrm{LE}\left(\mathrm{m}^{2}\right)$ \\
\hline$V_{U Q}^{*}$ & $R_{U Q}^{*}$ & 0.4171 \\
\hline$V^{*}$ & $R^{*}$ & 0.1949 \\
\hline
\end{tabular}

\section{CONCLUSIONS}

In this paper, we addressed the quantization-aware sensor selection problem for source localization in sensor networks. We proposed a sensor selection process equipped with the well known GBFOS. We demonstrated that the sensor selection problem could be solved by allocating rates to each sensor with a given cost and sensor locations and their relatives rates should be jointly optimized in order to find the best sensor selection solution.

\section{REFERENCES}

[1] J. C. Chen, K. Yao, and R. E. Hudson. Source localization and beamforming. IEEE Signal Processing Magazine, 19(2), March 2002.

[2] V. Isler and R. Bajcsy. The sensor selection problem for bounded uncertainty sensing models. IEEE Internationa Conference on Information Processing in Sensor Networks (IPSN), April 2005.

[3] Y. H. Kim. and A. Ortega. Quantizer design for source localization in sensor networks. IEEE International Conference on Acoustic, Speech, and Signal Processing (ICASSP), March 2005.

[4] Y. H. Kim. and A. Ortega. Maximun a posteriori (MAP)-based algorithm for distributed source localization using quantized acoustic sensor readings. IEEE International Conference on Acoustic, Speech, and Signal Processing (ICASSP), May 2006.

[5] D. Li and Y. H. Hu. Energy-based collaborative source localization using acoustic microsensor array. EURASIP Journal on Applied Signal Processing, pages 321-337, 2003.

[6] D. Li, K. D. Wong, Y. H. Hu, and A. M. Sayeed. Detection, classification and tracking of targets. IEEE Signal Processing Magazine, 19(2):17-29, March 2002.

[7] R. Niu and P. Varshney. Target location estimation in sensor networks with quantized data. appears in IEEE Transactions on Signal Processing.

[8] R. Niu and P. Varshney. Target location estimation in wireless sensor networks using binary data. In Proceedings of the 38th Annual Conference on Information Sciences and Systems, Princeton, NJ, March 2004.

[9] E. A. Riskin. Optimal bit allocation via the generalized BFOS algorithm. IEEE Trans. on Information Theory, 37:400-402, March 1991.

[10] H. Wang, K. Yao, G. Pottie, and D. Estrin. Entropy-based sensor selection heuristic for target localization. IEEE International Conference on Information Processing in Sensor Networks (IPSN), 2004.

[11] J.-J. Xiao, S. Cui, Z.-Q. Luo, and A. Goldsmith. Joint estimation in sensor networks under energy constraints. IEEE First Conference on Sensor and Ad Hoc Communications and Networks, October 2004

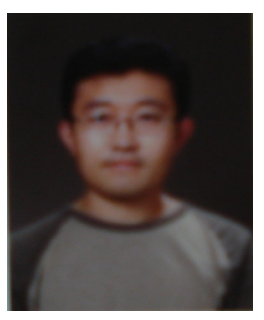

Yoon Hak Kim received his B.S. and M.S. degrees in electronic engineering from Yonsei University, Seoul, Korea in 1992 and 1994, respectively. He also received his Ph.D. degree in EE from University of Southern California in 2007. $\mathrm{He}$ is currently with System LSI division in Samsung Electronics Co., LTD. His research interests include distributed compression/ estimation in sensor networks with a focus on application-specific compression techniques, distributed source coding and image compression/enhancement algorithms. 